\title{
Nocardioides lentus sp. nov., isolated from an alkaline soil
}

Correspondence

Jung-Hoon Yoon

jhyoon@kribb.re.kr

\author{
Jung-Hoon Yoon, Choong-Hwan Lee and Tae-Kwang Oh \\ Korea Research Institute of Bioscience and Biotechnology (KRIBB), PO Box 115, Yusong, \\ Taejon, Korea
}

\begin{abstract}
Gram-positive, rod- or coccoid-shaped bacterial strains $\mathrm{KSL}-17^{\top}, \mathrm{KSL}-18$ and $\mathrm{KSL}-19$ were isolated from an alkaline soil in Korea. They were subjected to analysis using polyphasic taxonomy. Strains $\mathrm{KSL}-17^{\top}, \mathrm{KSL}-18$ and $\mathrm{KSL}-19$ grew optimally at $\mathrm{pH} 8.0$ and $28^{\circ} \mathrm{C}$ and in the presence of $0.5 \%(\mathrm{w} / \mathrm{v}) \mathrm{NaCl}$. They were characterized chemotaxonomically as having LL-2,6-diaminopimelic acid in the cell-wall peptidoglycan, $\mathrm{MK}-8\left(\mathrm{H}_{4}\right)$ as the predominant menaquinone and iso- $\mathrm{C}_{16: 0}$ as the major fatty acid. Their DNA G $+\mathrm{C}$ contents were in the range $74 \cdot 6-74 \cdot 8 \mathrm{~mol} \%$. Strains $\mathrm{KSL}-17^{\top}, \mathrm{KSL}-18$ and KSL-19 were identical in terms of their $16 \mathrm{~S}$ rRNA gene sequences and exhibited a mean level of DNA-DNA relatedness of 85-90\%. Phylogenetic analyses based on 16S rRNA gene sequences showed that strains $\mathrm{KSL}-17^{\top}, \mathrm{KSL}-18$ and $\mathrm{KSL}-19$ formed a distinct phylogenetic lineage within the genus Nocardioides. The levels of $16 \mathrm{~S}$ rRNA gene sequence similarity between the three strains and the type strains of Nocardioides species were in the range 92.6-95.2\%. On the basis of phenotypic, genetic and phylogenetic data, strains $\mathrm{KSL}-17^{\top}$ $\left(=\right.$ KCTC $19039^{\top}=$ DSM $\left.16315^{\top}\right)$, KSL-18 and KSL-19 should be classified as members of a novel species of the genus Nocardioides, for which the name Nocardioides lentus sp. nov. is proposed.
\end{abstract}

The genus Nocardioides was proposed by Prauser (1976), and, at the time of writing, the genus comprises 15 recognized species, including the recently described species Nocardioides alkalitolerans (Yoon et al., 2005a), Nocardioides kribbensis (Yoon et al., 2005b), Nocardioides oleivorans (Schippers et al., 2005) and Nocardioides dubius (Yoon et al., 2005c). In this study, we report the taxonomic characterization of three Nocardioides-like strains, KSL-17 ${ }^{\mathrm{T}}$, KSL-18 and KSL-19, which were isolated from an alkaline soil (approximately pH 9.0-10.0) collected in Kwangchun, Korea.

Strains KSL-17 ${ }^{\mathrm{T}}$, KSL-18 and KSL-19 were isolated by using the standard dilution plating technique at $30{ }^{\circ} \mathrm{C}$ on $10 \times$ diluted nutrient agar (Difco) with the $\mathrm{pH}$ adjusted to $9 \cdot 0$ using $\mathrm{Na}_{2} \mathrm{CO}_{3}$. To investigate their morphological, physiological and biochemical characteristics, strains KSL$17^{\mathrm{T}}$, KSL-18 and KSL-19 were routinely cultivated at $28^{\circ} \mathrm{C}$ on $2 \times$ diluted nutrient agar with the $\mathrm{pH}$ adjusted to $8 \cdot 0$. Morphological, physiological, cultural and biochemical

Published online ahead of print on 23 September 2005 as DOI 10.1099/ijs.0.63993-0.

The GenBank/EMBL/DDBJ accession numbers for the 16S rRNA gene sequences of strains $\mathrm{KSL}-17^{\top}, \mathrm{KSL}-18$ and $\mathrm{KSL}-19$ are DQ121389, DQ121390 and DQ121391, respectively.

A table giving the cellular fatty acid content of strains $\mathrm{KSL}-17^{\top}, \mathrm{KSL}$ 18 and KSL-19 is available as supplementary material in IJSEM Online. properties were examined as described by Yoon et al. (2005a). Growth in the absence of $\mathrm{NaCl}$ was investigated in trypticase soy broth prepared according to the formula of the Difco medium except that no $\mathrm{NaCl}$ was used. Growth at various $\mathrm{NaCl}$ concentrations $(0 \cdot 5 \%$, w/v, and $1 \cdot 0-7 \cdot 0 \%$, $\mathrm{w} / \mathrm{v}$, at intervals of $1.0 \%$ ) was investigated in trypticase soy broth (Difco). The $\mathrm{pH}$ range for growth was determined in $2 \times$ diluted nutrient broth (Difco) that was adjusted to various $\mathrm{pH}$ values (initial $\mathrm{pH} 4 \cdot 5-10 \cdot 5$ at intervals of $0.5 \mathrm{pH}$ units). The $\mathrm{pH}$ was adjusted, prior to sterilization, to various levels by the addition of $\mathrm{HCl}$ or $\mathrm{Na}_{2} \mathrm{CO}_{3}$. Cell biomass for DNA extraction and for the analyses of cell-wall and isoprenoid quinones was obtained by cultivation at $28^{\circ} \mathrm{C}$ in $2 \times$ diluted nutrient broth $(\mathrm{pH} 8 \cdot 0)$. Chemotaxonomic and molecular systematic studies were performed as described by Yoon et al. (2005a). The isomer type of the diamino acid in the cell-wall peptidoglycan was analysed using TLC according to the method described by Komagata \& Suzuki (1987). For fatty acid methyl ester analysis, cell mass from the three strains was harvested from $2 \times$ diluted nutrient agar ( $\mathrm{pH} 8 \cdot 0)$ after incubation for 10 days at $28^{\circ} \mathrm{C}$.

The morphological, cultural, physiological and biochemical characteristics of strains KSL-17 ${ }^{\mathrm{T}}$, KSL-18 and KSL-19 are given in the species description (see later) or are shown in Table 1. The $16 \mathrm{~S}$ rRNA gene sequences of strains KSL-17 $7^{\mathrm{T}}$, KSL-18 and KSL-19 were identical and each comprised $1491 \mathrm{nt}$, representing approximately $96 \%$ of the Escherichia 
Table 1. Differential phenotypic characteristics of Nocardioides lentus sp. nov. and related Nocardioides species

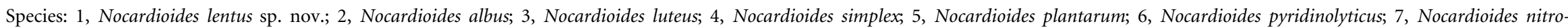

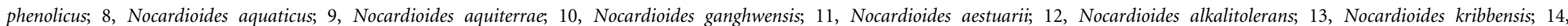

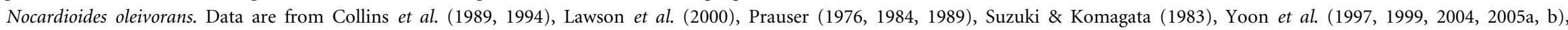

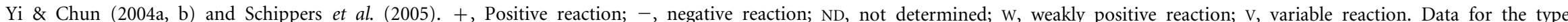

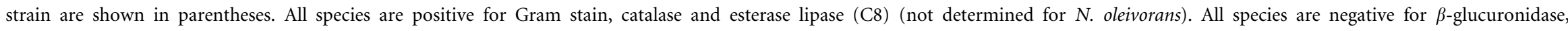
$N$-acetyl- $\beta$-glucosaminidase and $\alpha$-fucosidase (not determined for $N$. oleivorans).

\begin{tabular}{|c|c|c|c|c|c|c|c|c|c|c|c|c|c|c|}
\hline Characteristic & 1 & 2 & 3 & 4 & 5 & 6 & 7 & 8 & 9 & 10 & 11 & 12 & 13 & 14 \\
\hline Cell morphology & $\begin{array}{l}\text { Rods, } \\
\text { cocci }\end{array}$ & Hyphae & Hyphae & $\begin{array}{l}\text { Rods, } \\
\text { cocci }\end{array}$ & $\begin{array}{l}\text { Short rods, } \\
\text { cocci }\end{array}$ & $\begin{array}{l}\text { Rods } \\
\text { cocci }\end{array}$ & $\begin{array}{l}\text { Rods, } \\
\text { cocci }\end{array}$ & $\begin{array}{c}\text { Cocci, } \\
\text { short rods }\end{array}$ & $\begin{array}{l}\text { Rods, } \\
\text { cocci }\end{array}$ & Rods & Rods & $\begin{array}{l}\text { Rods, } \\
\text { cocci }\end{array}$ & $\begin{array}{c}\text { Short rods, } \\
\text { cocci }\end{array}$ & $\begin{array}{l}\text { Irregular } \\
\text { rods }\end{array}$ \\
\hline Cell size $(\mu \mathrm{m})$ & $\begin{array}{c}0 \cdot 4-0 \cdot 7 \times \\
1 \cdot 0-4 \cdot 5\end{array}$ & $0 \cdot 5-1 \cdot 0$ & $0 \cdot 5-1 \cdot 0$ & $\begin{array}{c}1 \cdot 0-1 \cdot 2 \times \\
1 \cdot 5-6 \cdot 0\end{array}$ & ND & $\begin{array}{c}0 \cdot 5-0 \cdot 6 \times \\
1 \cdot 2-1 \cdot 6\end{array}$ & $\begin{array}{c}0 \cdot 5-0 \cdot 8 \times \\
1 \cdot 0-3 \cdot 0\end{array}$ & $\begin{array}{c}0 \cdot 9-1 \cdot 0 \times \\
0 \cdot 9-1 \cdot 4\end{array}$ & $\begin{array}{c}0 \cdot 8-1 \cdot 0 \times \\
1 \cdot 7-2 \cdot 0\end{array}$ & $\begin{array}{c}0 \cdot 4-0 \cdot 5 \times \\
0 \cdot 9-4 \cdot 5\end{array}$ & $\begin{array}{c}0 \cdot 3-0 \cdot 4 \times \\
0 \cdot 9-2 \cdot 1\end{array}$ & $\begin{array}{c}0 \cdot 8-1 \cdot 0 \times \\
1 \cdot 5-2 \cdot 0\end{array}$ & $\begin{array}{c}0 \cdot 8-1 \cdot 0 \times \\
1 \cdot 5-2 \cdot 0\end{array}$ & $0 \cdot 3 \times \sim 1 \cdot 1$ \\
\hline Motility & - & - & - & + & - & + & + & - & + & - & - & - & - & - \\
\hline Colony colour ${ }^{\star}$ & Yellow & $\begin{array}{l}\text { Whitish to } \\
\text { faintly } \\
\text { brownish }\end{array}$ & $\begin{array}{l}\text { Yellow to } \\
\text { orange-yellow } \\
\text { or cream }\end{array}$ & $\begin{array}{l}\text { Yellowish } \\
\text { white }\end{array}$ & ND & Cream & $\begin{array}{l}\text { Yellowish } \\
\text { white }\end{array}$ & $\begin{array}{c}\text { Dull } \\
\text { orange }\end{array}$ & Cream & Ivory & Ivory & $\begin{array}{l}\text { Milky } \\
\text { white }\end{array}$ & Cream & Orange \\
\hline Optimal temp. $\left({ }^{\circ} \mathrm{C}\right)$ & 28 & 28 & 28 & $26-37$ & 25 & 35 & 30 & $16-26$ & 30 & 30 & 30 & $25-30$ & 30 & 30 \\
\hline Nitrate reduction & + & $(-)$ & - & $(-)$ & - & + & - & + & + & + & - & + & + & $\mathrm{ND}$ \\
\hline \multicolumn{15}{|l|}{ Hydrolysis of: } \\
\hline Aesculin & - & $(\mathrm{w})$ & + & $(+)$ & $\mathrm{w}$ & + & + & - & + & $\mathrm{W}$ & $\mathrm{W}$ & - & + & ND \\
\hline Casein & + & $(+)$ & + & + & $+\dagger$ & + & + & + & + & + & + & + & + & ND \\
\hline Starch & - & $(+)$ & + & (w) & - & + & + & $\mathrm{w}^{\dagger}$ & - & + & - & - & - & ND \\
\hline Gelatin & + & $(+)$ & + & + & + & + & + & + & + & + & + & $\mathrm{v}(+)$ & + & $\mathrm{ND}$ \\
\hline Tween 80 & + & $(+)$ & + & $(+)$ & + & - & + & + & + & + & + & + & + & $\mathrm{ND}$ \\
\hline Urea & - & $(-)$ & - & - & - & - & + & - & - & - & - & - & - & $\mathrm{ND}$ \\
\hline Hypoxanthine & - & $(+)$ & + & $(-)$ & - & - & - & - & - & - & - & - & - & ND \\
\hline Xanthine & - & $(+)$ & $-\dagger$ & $(-)$ & - & + & - & - & - & $\mathrm{W}$ & - & - & - & $\mathrm{ND}$ \\
\hline Tyrosine & $\mathrm{v}(+)$ & $(+)$ & + & $(+)$ & - & + & + & + & - & + & - & + & + & $\mathrm{ND}$ \\
\hline \multicolumn{15}{|c|}{$\begin{array}{l}\text { Utilization of substrates } \\
\text { as sole carbon and } \\
\text { energy sources }\end{array}$} \\
\hline L-Arabinose & $\mathrm{w}$ & + & + & - & - & - & - & - & - & + & - & + & - & - \\
\hline D-Cellobiose & + & $(+)$ & + & $(-)$ & + & + & - & - & + & + & + & + & + & + \\
\hline D-Fructose & - & + & $+\dagger$ & $(-)$ & + & + & + & + & + & + & + & - & - & + \\
\hline D-Galactose & + & $(+)$ & - & $(-)$ & - & + & - & $-\dagger$ & + & + & + & - & + & + \\
\hline D-Glucose & + & + & + & + & $+\dagger$ & + & + & + & + & + & + & - & + & + \\
\hline myo-Inositol & - & - & - & $(-)$ & - & + & - & $\mathrm{w}^{\dagger}$ & - & - & - & - & - & ND \\
\hline Lactose & $\mathrm{v}(+)$ & $(-)$ & - & $(-)$ & - & $+\dagger$ & - & - & + & + & $\mathrm{W}$ & - & - & ND \\
\hline Maltose & + & ND & ND & ND & + & + & - & $\mathrm{W}$ & + & ND & ND & $\mathrm{V}(-)$ & + & + \\
\hline D-Mannitol & + & + & + & - & - & $+\dagger$ & - & + & + & + & + & - & + & + \\
\hline
\end{tabular}


Table 1. cont.

\begin{tabular}{|c|c|c|c|c|c|c|c|c|c|c|c|c|c|c|}
\hline Characteristic & 1 & 2 & 3 & 4 & 5 & 6 & 7 & 8 & 9 & 10 & 11 & 12 & 13 & 14 \\
\hline D-Mannose & - & $(+)$ & + & $(-)$ & - & $+\dagger$ & $\mathrm{w} \dagger$ & - & + & + & - & - & - & + \\
\hline Melezitose & + & ND & $\mathrm{ND}$ & $\mathrm{ND}$ & + & ND & - & - & - & ND & ND & + & - & ND \\
\hline D-Melibiose & - & $\mathrm{ND}$ & $\mathrm{ND}$ & $\mathrm{ND}$ & ND & - & - & $\mathrm{W}$ & - & $\mathrm{ND}$ & $\mathrm{ND}$ & - & - & + \\
\hline D-Raffinose & + & $\mathrm{V}(-)$ & - & - & - & - & - & - & - & + & $\mathrm{W}$ & + & + & ND \\
\hline L-Rhamnose & + & + & $+\dagger$ & - & + & + & + & $+\dagger$ & $\mathrm{w} \dagger$ & - & - & - & + & + \\
\hline D-Ribose & - & $(-)$ & - & $(-)$ & - & + & + & - & $\mathrm{w} \dagger$ & - & - & - & + & - \\
\hline Sucrose & + & $\mathrm{v}(+\dagger)$ & $+\dagger$ & + & + & + & + & + & + & + & + & - & + & + \\
\hline D-Trehalose & + & ND & $\mathrm{ND}$ & ND & + & + & + & $\mathrm{W}$ & + & ND & ND & + & + & + \\
\hline D-Xylose & $\mathrm{v}(+)$ & + & + & - & + & + & + & $\mathrm{w} \dagger$ & + & + & + & - & - & $\mathrm{ND}$ \\
\hline \multicolumn{15}{|l|}{ API ZYM } \\
\hline Alkaline phosphatase & + & $(+)$ & $\mathrm{V}$ & $(+)$ & - & + & + & $\mathrm{V}$ & - & + & $\mathrm{w}$ & + & + & $\mathrm{ND}$ \\
\hline Esterase (C4) & + & $(+)$ & + & $(-)$ & + & - & - & $\mathrm{w}$ & - & $\mathrm{V}$ & + & + & + & ND \\
\hline Lipase (C14) & - & $(-)$ & - & $(-)$ & $\mathrm{W}$ & - & - & - & - & - & - & + & - & ND \\
\hline Leucine arylamidase & + & $(+)$ & + & $(+)$ & + & + & + & + & + & + & + & - & + & ND \\
\hline Valine arylamidase & - & $(-)$ & - & $(\mathrm{w})$ & $\mathrm{W}$ & $\mathrm{W}$ & + & $\mathrm{W}$ & - & + & + & - & - & ND \\
\hline Cystine arylamidase & - & $(-)$ & - & $(\mathrm{w})$ & + & - & $\mathrm{w}$ & $\mathrm{V}$ & $\mathrm{w}$ & $\mathrm{W}$ & - & - & - & ND \\
\hline Trypsin & - & $(+)$ & + & $(+)$ & - & + & + & $\mathrm{w}$ & + & - & + & - & - & ND \\
\hline$\alpha$-Chymotrypsin & - & $(-)$ & - & $(-)$ & - & - & - & - & - & - & + & - & + & ND \\
\hline Acid phosphatase & + & $(-)$ & - & (w) & $\mathrm{W}$ & + & + & + & + & $\mathrm{w}$ & $\mathrm{w}$ & + & + & $\mathrm{ND}$ \\
\hline $\begin{array}{l}\text { Naphthol-AS-BI- } \\
\text { phosphohydrolase }\end{array}$ & + & (w) & $\mathrm{V}$ & $(-)$ & + & + & + & - & + & - & $\mathrm{w}$ & $\mathrm{W}$ & + & ND \\
\hline$\alpha$-Galactosidase & - & $(-)$ & - & $(-)$ & - & - & - & - & - & + & - & - & - & ND \\
\hline$\beta$-Galactosidase & - & (v) & $\mathrm{V}$ & $(-)$ & - & - & - & - & - & + & + & - & $\mathrm{W}$ & ND \\
\hline$\alpha$-Glucosidase & - & $(+)$ & $\mathrm{V}$ & $(+)$ & + & + & + & + & + & + & + & + & + & ND \\
\hline$\beta$-Glucosidase & + & $(\mathrm{w})$ & - & (w) & + & - & $\mathrm{w}$ & - & $\mathrm{w}$ & - & - & - & - & $\mathrm{ND}$ \\
\hline$\alpha$-Mannosidase & - & $(-)$ & + & $(-)$ & - & - & - & - & - & - & - & - & - & $\mathrm{ND}$ \\
\hline $\begin{array}{l}\text { DNA G }+C \text { content } \\
(\mathrm{mol} \%)\end{array}$ & $74 \cdot 6-74 \cdot 8$ & 67 & 68 & $72-74$ & 69 & 73 & 71 & 69 & 73 & 72 & 70 & $72-74$ & $73-74$ & $\mathrm{ND}$ \\
\hline Isolation source & $\begin{array}{l}\text { Alkaline } \\
\text { soil }\end{array}$ & Soil & Soil & Soil & Herbage & $\begin{array}{l}\text { Oil-shale } \\
\text { column }\end{array}$ & $\begin{array}{l}\text { Industrial } \\
\text { wastewater }\end{array}$ & $\begin{array}{l}\text { Saline lake, } \\
\text { Antarctica }\end{array}$ & $\begin{array}{l}\text { Ground- } \\
\text { water }\end{array}$ & $\begin{array}{l}\text { Tidal } \\
\text { flat }\end{array}$ & $\begin{array}{l}\text { Tidal } \\
\text { flat }\end{array}$ & $\begin{array}{l}\text { Alkaline } \\
\text { soil }\end{array}$ & $\begin{array}{l}\text { Alkaline } \\
\text { soil }\end{array}$ & $\begin{array}{l}\text { Crude } \\
\text { oil }\end{array}$ \\
\hline
\end{tabular}

${ }^{\star}$ Differences may be caused by different cultivation conditions.

$\dagger$ Data from Prauser et al. (1976, 1984), Collins et al. (1994), Yoon et al. (1997, 1999, 2004) and Lawson et al. (2000); different results were reported by Yi \& Chun (2004a). 


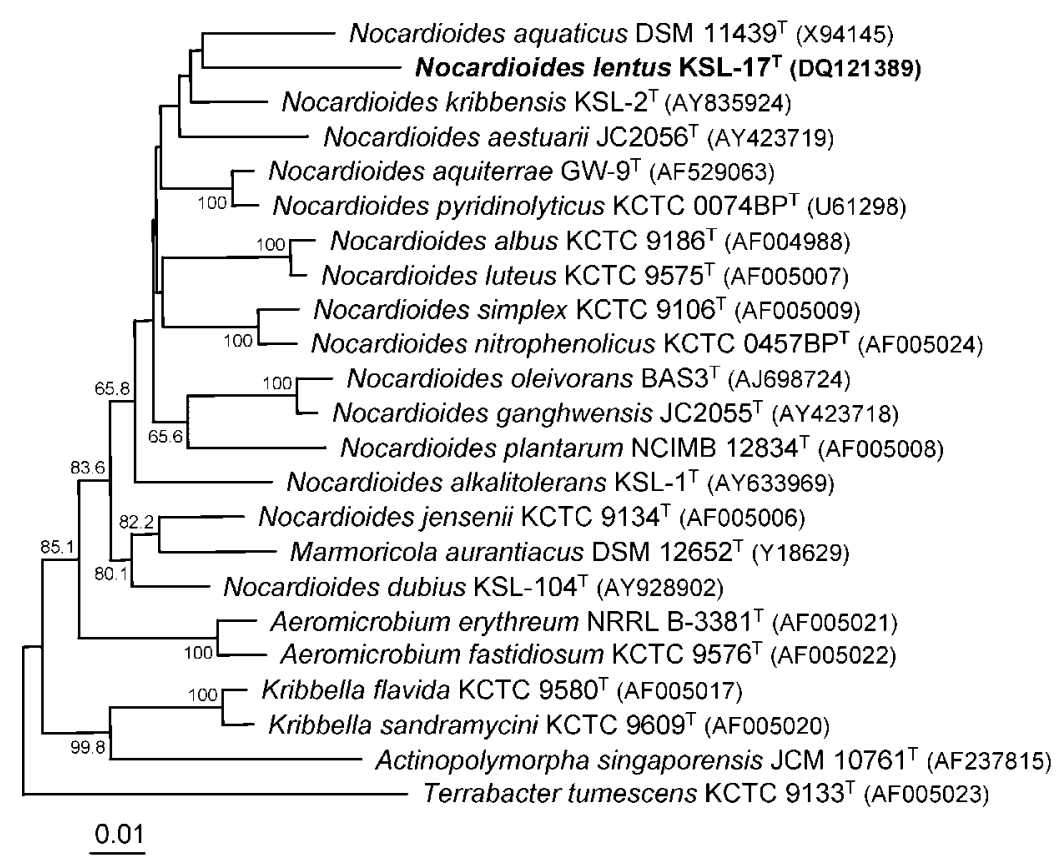

Fig. 1. Neighbour-joining phylogenetic tree, based on 16S rRNA gene sequences, showing the positions of strain $\mathrm{KSL}-17^{\top}$ and some other related taxa. Bootstrap values (expressed as percentages of 1000 replications) greater than $50 \%$ are shown at branch points. Bar, 0.01 substitutions per nucleotide position. coli 16S rRNA gene sequence. Comparative 16S rRNA gene sequence analyses showed that the three strains were phylogenetically affiliated to the genus Nocardioides (Fig. 1). In the phylogenetic trees based on the neighbour-joining, maximum-parsimony and maximum-likelihood algorithms, strains KSL- $17^{\mathrm{T}}$, KSL-18 and KSL-19 formed a distinct phylogenetic lineage within the radiation of the cluster comprising Nocardioides species (Fig. 1). Similarity values between the 16S rRNA gene sequences of the three strains and those of recognized Nocardioides species ranged from $92 \cdot 6 \%$ (Nocardioides albus KCTC $9186^{\mathrm{T}}$ ) to $95 \cdot 2 \%$ (N. kribbensis KSL-2 ${ }^{\mathrm{T}}$ ). Values for sequence similarity to other species included in the phylogenetic analysis were below $93 \cdot 3 \%$.

Strains KSL-17 ${ }^{\mathrm{T}}$, KSL-18 and KSL-19 contained LL-2,6diaminopimelic acid in the cell-wall peptidoglycan, the predominant menaquinone was $\mathrm{MK}-8\left(\mathrm{H}_{4}\right)$ and the fatty acid profiles comprised mainly iso- $\mathrm{C}_{16: 0}(60 \cdot 9-80 \cdot 9 \%)$, with smaller amounts of $\mathrm{C}_{17: 1} \omega 8 c(0 \cdot 5-6 \cdot 6 \%), 10$-methyl $\mathrm{C}_{17: 0}(1 \cdot 5-5 \cdot 8 \%)$, iso- $\mathrm{C}_{14: 0}(2 \cdot 3-6 \cdot 5 \%)$ and so on (see the supplementary table in IJSEM Online). The chemotaxonomic characteristics were consistent with the affiliation of the strains to the genus Nocardioides (Yoon et al., 1997, 2004, 2005a, b; Lawson et al., 2000; Urzì et al., 2000; Yi \& Chun, 2004a, b; Schippers et al., 2005). The DNA G + C contents of strains KSL- $17^{\mathrm{T}}$, KSL- 18 and KSL- 19 were $74 \cdot 8$, 74.7 and $74.6 \mathrm{~mol} \%$, respectively.

Phylogenetic analyses based on 16S rRNA gene sequences and chemotaxonomic data revealed that the three strains can be assigned to the genus Nocardioides (Miller et al., 1991; Tamura \& Yokota, 1994; Park et al., 1999; Urzì et al., 2000; Wang et al., 2001). The mean DNA-DNA relatedness levels between strains KSL-17 ${ }^{\mathrm{T}}$, KSL-18 and KSL-19 were
85-90\%, indicating that the three strains represent the same genomic species (Wayne et al., 1987). They also shared almost identical phenotypic properties. The levels of $16 \mathrm{~S}$ rRNA gene sequence similarity are low enough to exclude the possibility of assigning the three strains to a previously described Nocardioides species (Stackebrandt \& Goebel, 1994). The three strains were distinguishable from the other Nocardioides species on the basis of some phenotypic properties, as shown in Table 1. Therefore, on the basis of the data presented, strains KSL- $17^{\mathrm{T}}$, KSL-18 and KSL-19 should be classified in the genus Nocardioides as members of a novel species, for which the name Nocardioides lentus sp. nov. is proposed.

\section{Description of Nocardioides lentus sp. nov.}

Nocardioides lentus (len'tus. L. masc. adj. lentus slow, delayed, referring to slow growth).

Cells are aerobic, non-endospore-forming rods $(0 \cdot 4-0 \cdot 7 \times$ $1 \cdot 0-4 \cdot 5 \mu \mathrm{m})$ in the exponential phase of growth. Cells show rod-to-coccus morphogenesis from the early exponential phase to the stationary phase. Gram-positive but Gramvariable in old cultures. Colonies are circular, convex, smooth, glistening, yellow in colour and $0.5-1.0 \mathrm{~mm}$ in diameter after 10 days incubation on $2 \times$ diluted nutrient agar at $28^{\circ} \mathrm{C}$. Neither substrate mycelium nor aerial mycelium is formed. Growth occurs at 4 and $34^{\circ} \mathrm{C}$, but not at $35^{\circ} \mathrm{C}$. The optimal $\mathrm{pH}$ for growth is 8.0 ; growth occurs at $\mathrm{pH} 6.5$ and $9 \cdot 5$, but not at $\mathrm{pH} 6.0$ or $10 \cdot 0$. Growth occurs in the presence of $0-5 \%(\mathrm{w} / \mathrm{v}) \mathrm{NaCl}$, with an optimum at $0.5 \%(\mathrm{w} / \mathrm{v}) \mathrm{NaCl}$. Weakly positive for oxidase activity. Tweens 20, 40 and 60 are hydrolysed. D-Sorbitol is utilized, but adonitol is not. The cell-wall peptidoglycan contains LL-2,6-diaminopimelic acid as the diagnostic diamino acid. 
The predominant menaquinone is $\mathrm{MK}-8\left(\mathrm{H}_{4}\right)$. The major fatty acid is iso- $\mathrm{C}_{16: 0}$; 10-methyl fatty acids are present. The DNA G + C content is $74 \cdot 6-74 \cdot 8 \mathrm{~mol} \%$ (determined by HPLC). Other phenotypic characteristics are given in Table 1.

The type strain, KSL- $17^{\mathrm{T}}\left(=\right.$ KCTC $\left.19039^{\mathrm{T}}=\mathrm{DSM} 16315^{\mathrm{T}}\right)$, was isolated from an alkaline soil in Kwangchun, Korea. Reference strains are KSL-18 and KSL-19.

\section{Acknowledgements}

This work was supported by the 21C Frontier program of Microbial Genomics and Applications (grant MG05-0401-2-0) from the Ministry of Science and Technology (MOST) of the Republic of Korea.

\section{References}

Collins, M. D., Dorsch, M. \& Stackebrandt, E. (1989). Transfer of Pimelobacter tumescens to Terrabacter gen. nov. as Terrabacter tumescens comb. nov. and of Pimelobacter jensenii to Nocardioides as Nocardioides jensenii comb. nov. Int J Syst Bacteriol 39, 1-6.

Collins, M. D., Cockcroft, S. \& Wallbanks, S. (1994). Phylogenetic analysis of a new LL-diaminopimelic acid-containing coryneform bacterium from herbage, Nocardioides plantarum sp. nov. Int J Syst Bacteriol 44, 523-526.

Komagata, K. \& Suzuki, K. (1987). Lipids and cell-wall analysis in bacterial systematics. Methods Microbiol 19, 161-203.

Lawson, P. A., Collins, M. D., Schumann, P., Tindall, B. J., Hirsch, P. \& Labrenz, M. (2000). New LL-diaminopimelic acid-containing actinomycetes from hypersaline, heliothermal and meromictic Antarctic Ekho Lake: Nocardioides aquaticus sp. nov. and Friedmanniella lacustris sp. nov. Syst Appl Microbiol 23, 219-229.

Miller, E. S., Woese, C. R. \& Brenner, S. (1991). Description of the erythromycin-producing bacterium Arthrobacter sp. strain NRRL B-3381 as Aeromicrobium erythreum gen. nov., sp. nov. Int J Syst Bacteriol 41, 363-368.

Park, Y.-H., Yoon, J.-H., Shin, Y. K., Suzuki, K.-I., Kudo, T., Seino, A., Kim, H.-J., Lee, J.-S. \& Lee, S. T. (1999). Classification of 'Nocardioides fulvus' IFO 14399 and Nocardioides sp. ATCC 39419 in Kribbella gen. nov., as Kribbella flavida sp. nov. and Kribbella sandramycini sp. nov. Int J Syst Bacteriol 49, 743-752.

Prauser, H. (1976). Nocardioides, a new genus of the order Actinomycetales. Int J Syst Bacteriol 26, 58-65.

Prauser, H. (1984). Nocardioides luteus spec. nov. Z Allg Microbiol 24, 647-648.

Prauser, H. (1989). Genus Nocardioides Prauser 1976. In Bergey's Manual of Systematic Bacteriology, vol. 4, pp. 2371-2375. Edited by S. T. Williams, M. E. Sharpe \& J. G. Holt. Baltimore: Williams \& Wilkins.
Schippers, A., Schumann, P. \& Spröer, C. (2005). Nocardioides oleivorans sp. nov., a novel crude oil-degrading bacterium. Int J Syst Evol Microbiol 55, 1501-1504.

Stackebrandt, E. \& Goebel, B. M. (1994). Taxonomic note: a place for DNA-DNA reassociation and $16 \mathrm{~S}$ rRNA sequence analysis in the present species definition in bacteriology. Int J Syst Bacteriol 44, 846-849.

Suzuki, K. \& Komagata, K. (1983). Pimelobacter gen. nov., a new genus of coryneform bacteria with LL-diaminopimelic acid in the cell wall. J Gen Appl Microbiol 29, 59-71.

Tamura, T. \& Yokota, A. (1994). Transfer of Nocardioides fastidiosa Collins and Stackebrandt 1989 to the genus Aeromicrobium as Aeromicrobium fastidiosum comb. nov. Int J Syst Bacteriol 44, 608-611.

Urzi, C., Salamone, P., Schumann, P. \& Stackebrandt, E. (2000). Marmoricola aurantiacus gen. nov., sp. nov., a coccoid member of the family Nocardioidaceae isolated from a marble statue. Int J Syst Evol Microbiol 50, 529-536.

Wang, Y. M., Zhang, Z. S., Xu, X. L., Ruan, J. S. \& Wang, Y. (2001). Actinopolymorpha singaporensis gen. nov., sp. nov., a novel actinomycete from the tropical rainforest of Singapore. Int J Syst Evol Microbiol 51, 467-473.

Wayne, L. G., Brenner, D. J., Colwell, R. R. \& 9 other authors (1987). International Committee on Systematic Bacteriology. Report of the ad hoc committee on reconciliation of approaches to bacterial systematics. Int J Syst Bacteriol 37, 463-464.

Yi, H. \& Chun, J. (2004a). Nocardioides ganghwensis sp. nov., isolated from tidal flat sediment. Int J Syst Evol Microbiol 54, 1295-1299.

Yi, H. \& Chun, J. (2004b). Nocardioides aestuarii sp. nov., isolated from tidal flat sediment. Int J Syst Evol Microbiol 54, 2151-2154.

Yoon, J.-H., Rhee, S.-K., Lee, J.-S., Park, Y.-H. \& Lee, S. T. (1997). Nocardioides pyridinolyticus sp. nov., a pyridine-degrading bacterium isolated from the oxic zone of an oil shale column. Int J Syst Bacteriol 47, 933-938.

Yoon, J.-H., Cho, Y.-G., Lee, S. T., Suzuki, K.-I., Nakase, T. \& Park, Y.-H. (1999). Nocardioides nitrophenolicus sp. nov., a p-nitrophenoldegrading bacterium. Int J Syst Bacteriol 49, 675-680.

Yoon, J.-H., Kim, I.-G., Kang, K. H., Oh, T.-K. \& Park, Y.-H. (2004). Nocardioides aquiterrae sp. nov., isolated from groundwater in Korea. Int J Syst Evol Microbiol 54, 71-75.

Yoon, J.-H., Kim, I.-G., Lee, M.-H., Lee, C.-H. \& Oh, T.-K. (2005a). Nocardioides alkalitolerans sp. nov., isolated from an alkaline serpentinite soil in Korea. Int J Syst Evol Microbiol 55, 809-814.

Yoon, J.-H., Kim, I.-G., Lee, M.-H. \& Oh, T.-K. (2005b). Nocardioides kribbensis sp. nov., isolated from an alkaline soil. Int J Syst Evol Microbiol 55, 1611-1614.

Yoon, J.-H., Lee, C.-H. \& Oh, T.-K. (2005c). Nocardioides dubius sp. nov., isolated from an alkaline soil. Int J Syst Evol Microbiol 55, 2209-2212. 